\title{
Regular monitoring with stool chart prevents constipation, urinary retention and delirium in elderly patients: an audit leading to clinical effectiveness, efficiency and patient centredness
}

\author{
Authors: Tun Zan Maung and Kanwaljit Singh
}

\section{Introduction}

Monitoring patients' bowel habits is very important in geriatric wards. Constipation in elderly patients may result in urinary retention, delirium and bowel obstruction. However, there was a concern that bowel monitoring might have been missed and that documentation in stool charts was occasionally not completed in busy medical wards. We carried out the study to assess the quality of bowel monitoring with the Bristol stool chart. The standard is that every elderly patient should have bowel monitoring with the stool chart every day. The stool chart must be fully documented. The compliance must be $100 \%$.

\section{Methods}

Inclusion criteria: every elderly patient admitted to Frailty Unit of Good Hope Hospital.

The first study was performed in November 2017. After the intervention, the audit was repeated in January 2018 to reassess the situation. We looked into three domains: whether it was documented, the quality of documentation and medical review of the chart.

Data was collected retrospectively from nursing and medical notes.

As intervention, a small group training of nursing staff was arranged to improve stool chart monitoring in elderly patients. Audit was presented at the weekly elderly care meeting.

\section{Results and discussion}

The first audit showed compliance of $85 \%$ in documentation, $75 \%$ in eligibility and $90 \%$ in medical review. We also noted one patient got urinary retention due to constipation and needed to be catheterised. Another had delirium due to constipation. During the study, we found out that many nurses were not aware of the importance of proper stool chart monitoring in elderly patients.
After the intervention, we could see an improvement with compliance of $91 \%$ in documentation, $93 \%$ in eligible documentation and $91 \%$ in medical review. No patient had urinary retention and delirium due to constipation.

\section{Conclusion}

Although we could see an improvement in documentation, we still need to continue educating nursing staff and junior doctors to get $100 \%$ compliance of stool chart monitoring. We are also planning to repeat the audit in regular cycles in the future.

\section{Conflict of interest statement}

None declared. 